\title{
Murburn precepts for the light reaction of oxygenic photosynthesis
}

\author{
Kelath Murali Manoj ${ }^{1}$ *, N M Bazhin ${ }^{2}$, Abhinav Parashar ${ }^{3}$,Daniel Andrew Gideon ${ }^{l}$, Vivian David Jacob ${ }^{1}$, \\ Deepak Haarith ${ }^{4}$ Afsal Manekkathodi ${ }^{1}$ \\ *Corresponding author, ${ }^{1}$ Satyamjayatu: The Science \& Ethics Foundation, \\ Kulappully, Shoranur-2 (PO), Palakkad District, Kerala State, India-679122. \\ Email: satyamjayatu@yahoo.com \\ ${ }^{2}$ Institute of Chemical Kinetics and Combustion \\ Russian Academy of Sciences, St. Institutskaya 3, 630090 Novosibirsk, Russia. \\ ${ }^{3}$ Department of Biotechnology, \\ Vignan's Foundation for Science, Technology \& Research, Vadlamudi, Guntur, India-522213. \\ ${ }^{4}$ Department of Plant Pathology, University of Minnesota, \\ St. Paul, MN 55108, USA
}

\begin{abstract}
Robert Emerson's original observation (1957) that "oxygenesis occurs even with farred light excitation of Photosystem I" is incompatible with the extant Kok-Joliot cycle's foundation that "photolysis occurs only at red-light stimulated Photosystem II harboring MnComplex". Further, the Z-scheme of electron transfer cannot account for Emerson's observations of enhanced oxygenesis by simultaneous excitation of the two photosystems with both red and far-red light because serially connected components would surely increase systemic resistance to flow of charges, impeding the overall electron transfer process from water to $\mathrm{NADP}^{+}$. To address such discrepancies, we propose that the photo-excitation of various pigments leads to the formation of aquated electrons $\left(\mathrm{e}_{\mathrm{aq}}\right)$ and diffusible reactive oxygen species (DROS) in milieu, which are stabilized by a pool of redox-active elements within chloroplasts. Subsequently, the ' $\mathrm{e}_{\mathrm{aq}}+\mathrm{DROS}$ ' pool is utilized and routed via disordered and parallel reactions by the 'photosystem switches' for NADP reduction, $\mathrm{O}_{2}$ liberation and ADP phosphorylation. The stochastic 'murburn' model is thermodynamically/kinetically favorable and evidenced by the identification of multiple ADP-binding sites on PS II/Cytochrome $b_{6} f$, and structure/distribution of the concerned proteins, complexes and pigments. The new model also explains the observed synergy in functioning of photosystems and plants' photosynthetic spectral range of 400-700 nm.
\end{abstract}

Keywords: photosynthesis; photoloysis; photophosphorylation; light reaction; Z-scheme; KokJoliot cycle; murburn concept; chloroplast; electron transfer; ATP synthesis; oxygen-evolution; reactive oxygen species (ROS); $Q$ cycle; 
Emerson's findings and the extant explanations for harvesting of sunlight by plants: Light reaction of photosynthesis generates the gaseous oxygen used by aerobes for cellular respiration and forms the primary source of ATP and NADPH plants use for fixing $\mathrm{CO}_{2}$, thereby sustaining the vast majority of life forms on planet earth. The 'Emerson enhancement effect' (Figure 1A) experiment (1) is a key forbearer of the concept that two photosystems work in tandem within plant chloroplasts. Mitchell-Boyer's “chemiosmotic rotary ATP synthesis” (CRAS) and HillBendall's multistep "Z-scheme of electron transport" (or ZS; including the photosystems' discharge-charge cycles, Q Cycle for quinols at Cytochrome $b_{6} f$ and the Mn-Complex mediated Kok-Joliot cycle or KJC for oxygenesis) are the currently standing explanations for the light reaction of oxygenic photosynthesis (Figure 1B), also known as PhotolysisPhotophosphorylation (Pl-Pp) (2). We find that the ZS-KJC paradigm is incompatible with key aspects of Robert Emerson's findings [reproducible in diverse photosynthetic systems (3)]. As per the current model, Photosystem I (PS I or P700, excited at far-red light) cannot photolyze water because it lacks the water-splitting or oxygen-evolving complex (WSC/OEC) or MnComplex. Then, it is inexplicable why Emerson observed that oxygen evolved in the instance when the system was exposed to only far-red light. Therefore, the extant KJC model's doctrine that oxygen evolution occurs only at WSC of Photosystem II (PS II or P680, excited at red light) seems misplaced. From another angle- without PS II, PSI would be dysfunctional because it cannot directly drain electrons from water. Therefore, the ZS-KJC explanation does not afford independent functioning and evolution of PS I. Further, Z-scheme proposes an elaborate route for the travel of electrons derived by photolysis of water, via the photosystems and redox proteins, to the ultimate destination of $\mathrm{NADP}^{+}$. Figure $1 \mathrm{C}-\mathrm{E}$ analyzes the oxygen yield ( $\approx$ electron flow) by a comparison of 'electron transport' with fluid flow through pipelines. In this case, the combined output of any number of elements connected in series is limited by the component with the slowest flow rate within the system. Therefore, we infer that ZS-KJC's serial connectivity of components (with photolysis occurring only at PS II) cannot explain the enhancement of photosynthesis obtained with the simultaneous illumination of PS I and PS II. Quite contrastingly, a parallel scheme of connectivity affords a higher total flow than the individual components working independently. This inference is further corroborated by a comparison of the system with electric circuits (Figure 1F-H, explained in detail in an ensuing section). Therefore, Z-scheme fails to explain the synergy of PS I+PS II. 
The search/approach for explanations of PI-Pp: Historically, several stalwart scientists like Edward Slater (pioneer in the field and long-term editor of BBA-Bioenergetics), Britton Chance (inventor of stopped-flow enzymology), Robert Williams (Irving-Williams series fame), Walter Wainio (pioneer of cytochrome oxidase structure-function) and Gilbert Ling (inventor of LingGerard electrode) had questioned Peter Mitchell's proposal that "electron transfers in mitochondrial/chloroplast membrane proteins is coupled to phosphorylation of ADP via a proton motive force (pmf)" (4). Otto Warburg and Robert Emerson, the famed teacher-disciple duo of Pl-Pp research, had unsettled disagreements on the number of photons required to make one molecule of $\mathrm{O}_{2}(5)$ and it is no surprise that the Editorial of Science (21 ${ }^{\text {st }}$ June, 2013, page 1373) had noted that- "It is unclear how energy absorbed by pigments in antenna proteins is transferred to the central site of chemical catalysis during photosynthesis." Daniel Arnon, the renowned plant physiologist (who originally mooted the concepts of cyclic and noncyclic photophosphorylation) had challenged the Z-scheme with valid arguments/evidence and proposed an independent functioning of PS I \& II (6), which was sidelined by skeptical peers (7). The above instances are quoted to demonstrate that Pl-Pp mechanism is a highly daunting conundrum because of the complexities involved in- (i) light's interaction with the numerous pigments arranged in diverse configurations within the intricate chloroplast architecture, (ii) unraveling the logic of energy transduction and electron transfers among the discretely distributed proteins on the thylakoid membrane/lumen and stroma, (iii) pin-pointing the reactions and site(s) of oxygen evolution, and (iv) understanding the coupling of the three processes above to the modality of ATP synthesis.

Murburn concept (from "mured burning", implying closed oxidation) is an evidence-based redox mechanistic paradigm that professes ubiquitous/stochastic interactive redox equilibriums among molecules, unbound ions and radicals (8). Murburn schemes advocate that diffusible reactive (oxygen) species (DRS/DROS/ROS; exemplified by $\mathrm{O}_{2}{ }^{*}-\mathrm{OH}^{-}$ions, $\mathrm{H}_{2} \mathrm{O}_{2} /{ }^{1} \mathrm{O}_{2}$ molecules, *OH radical, $\mathrm{e}_{\mathrm{aq}}$, etc.) serve as crucial elements in metabolic electron/moiety transfer processes (8). We used the insightful structural information on membrane redox proteins to advocate a murburn paradigm in heme/flavin enzymology (9-11), xenobiotic metabolism (mXM) (12), unusual physiological dose responses (13), mitochondrial oxidative phosphorylation (mOxPhos) 
and thermogenesis (14-17). The acclaimed CRAS paradigm of both mOxPhos and Pl-Pp encompasses electron transport chain (ETC) proteins serving as proton pumps to generate pmf, which is harnessed by a rotary ATP synthase function (2). The mOxPhos toxin of cyanide (CN) is also an established inhibitor of Pl-Pp system and its components (18-22). Another mechanistic commonality is that uncouplers (substituted phenolics like dinitrophenol) disrupt ATP synthesis in both the membrane redox systems (2). In this regard, we pointed out that substituted phenolics critically affect electron transfers even in $\mathrm{mXM}$ and since this system does not involve any "harnessing of pmf", such uncouplers are more likely to serve as interfacial proton/DROS modulators (and not trans-membrane proton-shuttlers) $(12-17,23,24)$. The commonality in the nature of catalysts (porphyrin/flavin/FeS-bound proteins working at phospholipid interfaces), cofactors (nicotinamide nucleotides), and products (DROS $+\mathrm{Heat}+\mathrm{H}_{2} \mathrm{O} / \mathrm{O}_{2}$ ) is also evident across the systems. The molecular species of oxygen, DROS and water are also ubiquitous in the microsomal, mitochondrial and thylakoid systems, and their roles cannot be restricted to any unique locus, per se. Most importantly, these membrane-interface reaction systems show nonintegral and variable stoichiometry, and a 'thermodynamic pull' exerted by the presence of 'substrates/uncouplers' (12-17,23-25). These similarities and parallels in the phenomenology of mXM, mOxPhos and Pl-Pp systems (Table 1) argue for " a stochastic paradigm involving DROS as delocalized operative/transducing elements" in the three redox routines. Recent theoretical and experimental (in vitro, and in situ) demonstrations of DROS-mediated electron/moiety transfers in xenobiotic hydroxylation, ADP phosphorylation, etc. (12-17,25) consolidate the murburn purview. Further, the following two quantitative facts remain at odds with the extant explanations: (i) By virtue of being micro-dimensioned organelles, mitochondria and chloroplasts are practically "aprotic" systems that have only $\sim 10^{1}$ 'free protons' (which cannot cater to $\sim 10^{5}$ redox proteins!). Therefore, it is a quantitatively untenable concept that proteins pump protons in such organelles (4,14-17). (ii) Several steps within the purported scheme (electron transfer coupled to proton pump) cannot occur with kinetic and thermodynamic viability via Marcus outer sphere mechanism (4,6). (iii) The Complex V sponsored equilibrium driven "electro-mechanical" ATP synthesis is a low probability event that could only give picomolar levels of ATP in physiological realms (16,17). Furthermore, the recent elucidation of a murburn-based mechanism of CN-toxicity in various redox systems (24-28) warrants a revisit of the explanations for Pl-Pp, as seen from the murburn perspective. Herein, we employ known 
small-molecule docking methodologies and visualization of protein structures (16) for understanding structure-function correlations and use transformed Gibbs free energy value calculations (29) to the elucidate the chemico-physical and biological principles governing Pl-Pp.

The 'connectivity' of redox-active components of Pl-Pp: Though the electron flow in Pl-Pp is noncyclic (the electrons derived from water gets stored in NADPH), it is possible to relate the working logic of such electron movement with the more familiar electrical/electronic circuitry systems. This can be envisaged by a steady-state wherein the light driven photolysis of water (leading to NADPH formation) is akin to the cyclic photosynthesis in prokaryotes using only PS I. As shown in such an analogous system in Figure $1 \mathrm{~F}-\mathrm{H}$, while light excitation is the powering principle that generates the emf, other 'material' components of Z-scheme can be deemed as electron-conducting and/or electron-discharging elements. The latter facets are the respective electronic principles of conductance (inverse of the resistance to flow of charges, R) and capacitance ( $\mathrm{C}$, the ability to store and release charges). In such a system, the overall charge flow from water to $\mathrm{NADP}^{+}$would determine the oxygen evolution $(\approx$ water lysis $)$. That is, higher the electron flow (current), greater is the oxygen evolution amount/rate. To obtain a higher electron flow, lower total resistance (or high conductance) and higher total capacitance (high charge storing/releasing ability) are desired. Theoretically, the components could work in three combinations, as shown in Figure 1F-H: (a) independently, without any inter-connectivity or synergism, (b) connected via series (as in the ZS), and (c) functioning via a parallel modality, using a common electron pool. If the elements of the Pl-Pp were connected in series, it would only increase the total resistance (or, decrease conductance) and lower capacitance. Within the equations given in Figure $1 \mathrm{G} \& 1 \mathrm{H}$, assigning arbitrary positive values for resistance/capacitance verifies that an independent and parallel functioning of PS I and PS II could explain the synergy (or greater electron flow or higher oxygen yield) obtained with the combined illumination of the two photosystems. To explain the higher efficiency of the Pl-Pp system by the simultaneous illumination with red $(680 \mathrm{~nm})$ and far-red $(700 \mathrm{~nm})$ light, we must also acknowledge the presence of multiple "non-specific electron reservoirs/buffers and connectivity networks" within, similar to the murburn schemes proposed for mXM (12) or mOxPhos $(16,17)$. We propose that such a feature would be enabled by various photo-active pigments (such as chlorophyll a/b, luteins, zeaxanthins, carotenoids, lycopenes, etc.) discharging electrons into the "reservoir" 
(Figures $1 \& 2$ ). This is the reason why- (i) a 'priming flash' is needed for the dark-acclimatized photosynthetic system in the Kok-Joliot experiments $(2,30)$, (ii) the number of photons required for the release of one molecule of oxygen was/is debated (5).

The logic, thermodynamics and kinetics of PI-Pp: As per the extant paradigm (2), PS II serves to trap light energy and Cyt. $b_{6} f$ helps to recycle the quinols formed at PS II; and both proteins 'pump protons' to generate a proton-gradient across the thylakoid membrane. The nanoscale dimensions of thylakoids and the alkaline $\mathrm{pH}$ value of $7.8-8.2(31,32)$ discount the viability of proton pumps $(4,14)$. In such scenario, an additional $\sim 101 \mathrm{~kJ} / \mathrm{mol}$ would be required $[79.1 \mathrm{~kJ} / \mathrm{mol}$ for heterolytic dissociation of water (33) and $22 \mathrm{~kJ} / \mathrm{mol}$ for the process of pumping out protons to generate $\operatorname{pmf}(2,4)]$. This would make the CRAS proposal energetically nonviable. Further, the Z-scheme does not offer reasons why- (i) $~ 5 \mathrm{~nm}$ lumen-ward extensions are found in PS II (34) and Cyt. $b_{6} f(35)$ (Figure 3), (ii) certain redox centers within the ETC are located far apart with little or unfavorable redox gradients, (iii) the numerous "non-route" redox-active pigments located in the membrane or DRS do not shunt the purported electron-circuitry, (iv) redox transfers are made by taking/giving one or two or four electrons, and (v) the electron transfers are spatially demarcated and span across three phases. For example- the current awareness on PS II structure reveals that while the reaction center of PS II is embedded in the trans-membrane region, the MnComplex is located in the lumen side, >2 nm away (36). After photoelectric emission, it is unknown how the reaction center would command a suitable response from the MnComplex. Or, since the MnComplex is not photo-active on its own merit, there is no known logic for a spontaneous one-electron supply from the source of " $2 \mathrm{H}_{2} \mathrm{O}-\mathrm{MnComplex"} \mathrm{via} \mathrm{a} \mathrm{four-}$ electron KJC. It is also unforeseeable how the MnComplex could hold on to the various species formed by electron/proton or hydrogen atom abstraction from two water molecules. This predicament is quite similar to the mechanistic perception of mitochondrial cytochrome oxidase holding on to a molecule of oxygen, waiting for four protons and four electrons, to produce two molecules of water and "pumping protons" in the redox process (4). From the redrawn Z-scheme in Figure 1B, the chinks in electron transfer are clearly evident. PS I or II can only accept electrons when they are positively charged (a highly reactive transient phase) and it is not clear how the electron supply is deterministically timed in these systems. The bulky semiquinones and quinols $\left(\mathrm{PQ}^{*-}\right.$ and $\mathrm{PQH}_{2}$ ) would have low mobilities within the lipid phase owing to their head's 
polarity, extra bulk/length and isoprenoid nature of the tail. The tetra-molecular Q-cycle is a lowprobability scheme that seeks a millisecond-scale binding of three different molecular species (quinone, quinol and oxidized plastocyanin) to three distinct loci of Cyt. $b_{f} f$. In vitro, the experimental equilibrium constant $\left(\mathrm{K}_{\mathrm{eq}}\right)$ for plastocyanin's $(\mathrm{PC})$ interaction with Cyt. $b_{6} f$ is only $2 \times 10^{0}(37)$, which is very low compared to the $1 \mathrm{e}$ or $2 \mathrm{e}$ bimolecular murburn reactions of semiquinone+DROS with $\mathrm{K}_{\mathrm{eq}}$ values of $10^{15}$ to $10^{31}$ and/or Keq values of $10^{10}$ to $10^{26}$ for recycling of quinol with oxygen [as shown in Table 2 of (33)]. The rate constant $(k)$ values of $10^{7}$ to $10^{8} \mathrm{M}^{-1} \mathrm{~s}^{-1}$ were noted (37) for the oxidation of Cyt. $b_{6} f$ and reduction of PS I by the mobile $\mathrm{Cu}$-protein. Owing to the requirement of protons at the membrane-milieu interface (physiological concentration $\sim 10^{-8} \mathrm{M}$ in chloropasts and practically unavailable in a resting thylakoid), levying a limiting substrate constraint of at least $10^{-6} \mathrm{M}$ gives a timescale of $10^{-1}$ to $10^{-2} \mathrm{~s}$ for $\mathrm{PC}$ mediated reactions at Cyt. $b_{6} f$ and PS I, which cannot be reconciled with- (i) the $10^{-12} \mathrm{~s}$ timescales for excitation and electron-discharge of the two reaction centers in the middle of the sequence, (ii) reactions of $10^{-6}$ and $10^{-9} \mathrm{~s}$ timescales at several pitstops/junctions, and (iii) oxygen liberation at the beginning or NADP reduction at the end of the sequence, both of which occur at $\sim 10^{-3} \mathrm{~s}$ timescales. Therefore, the serial linking of Cyt. $b_{6} f$ with PS II and PS I does not afford temporal viability. Q-cycle also seeks a thermodynamically inexplicable outcome that as the quinol gets oxidized by PC, electrons must also flow from the quinol to quinone within a purported circuitry where redox pitstops are separated by distances $>10 \AA$. Therefore, the proposal that "the relatively bulky plastoquinone (PQ, $0.75 \mathrm{KD})$ undertakes a deterministic journey in the membrane phase from PS II located at deep loci within the grana to Cyt. $b_{6} f$ located in peripheral thylakoids, and PC ( $11 \mathrm{KD})$ relays the electron from thereon via the aqueous phase to PS I" makes little kinetic logic. Across the known structures, the minimal $\mathrm{Cu}$ Fe distance in PC-Cyt. $f$ complex would be $>1 \mathrm{~nm}$ and $\mathrm{Cu}-\mathrm{Mg}$ in $\mathrm{RC}$ donor chlorophyll distance in PC-PS I complex would be >2 nm. Conservation of a small hydrophobic patch and approximate redox potential in PC (36) cannot overcome the fact that surface residues and $\mathrm{Cu}$ coordination sphere vary significantly across various species. This is not expected 'evolutionarily' if PC served as the protein-protein electron transfer liaison between the photosystems. Most importantly, as demonstrated for the mitochondrial Q-cycle at Complex III or Cyt. $b c_{1}(33)$, the deterministic Q-cycle at thylakoids' Cyt. $b_{6} f$ would not afford adequate energetic yield to enable proton-pumping and ATP-synthesis. 
The bond energetics calculation shows that the energy needed for breaking four O-H bonds (each of $\sim 464 \mathrm{~kJ} / \mathrm{mol}$ ) from the two molecules of water is $\sim 1856 \mathrm{~kJ} / \mathrm{mol}$. Analyzing from a reaction energetics perspective, if the photolysis occurred via an overall 2e paradigm of the CRAS-ZS model, the minimal nett transformed Gibbs' free energy that would entail is $(17,33,39)$ -

$2 \mathrm{H}_{2} \mathrm{O}+2 \mathrm{Q} \rightarrow 2 \mathrm{QH}_{2}+\mathrm{O}_{2}$ (PS II reaction)

$2 \mathrm{QH}_{2}+4 \mathrm{PC} \rightarrow 4 \mathrm{PCH}+2 \mathrm{Q}\left(\right.$ Cyt. $b_{6} f$ reaction $)$

$4 \mathrm{PCH}+2 \mathrm{NADP}^{+} \rightarrow 2 \mathrm{NADPH}+2 \mathrm{H}^{+}+4 \mathrm{PC}(\mathrm{PS} \mathrm{I}+\mathrm{FNR}$ reaction $)$

$2 \mathrm{H}_{2} \mathrm{O}+2 \mathrm{NADP}^{+} \rightarrow \mathrm{O}_{2}+2 \mathrm{NADPH}+2 \mathrm{H}^{+}\left(\mathrm{ZS}-\mathrm{ETC}: \Delta_{\mathrm{r}} G_{\text {aq }}^{\mathrm{o}}=1354.9 \mathrm{~kJ} / \mathrm{mol}\right)$

$3 \mathrm{ADPOH}+3 \mathrm{POH} \rightarrow 3 \mathrm{ADPOP}+3 \mathrm{H}_{2} \mathrm{O}\left(\mathrm{CRAS}: \Delta_{\mathrm{r}} G_{\mathrm{aq}}^{\mathrm{o}}=108.9 \mathrm{~kJ} / \mathrm{mol}\right)$

$2 \mathrm{NADP}^{+}+3 \mathrm{ADPOH}+3 \mathrm{POH} \rightarrow \mathrm{O}_{2}+2 \mathrm{NADPH}+2 \mathrm{H}^{+}+3 \mathrm{ADPOP}+\mathrm{H}_{2} \mathrm{O}\left(\right.$ Overall $\left.\Delta_{\mathrm{r}} G_{\mathrm{aq}}^{\mathrm{o}}=1463.8 \mathrm{~kJ} / \mathrm{mol}\right)$

However, the input of 4 einsteins each of $680 \mathrm{~nm}$ and $700 \mathrm{~nm}$ photons give only $1387.3 \mathrm{~kJ} / \mathrm{mol}$. Therefore, the extant paradigm advocates that the plant photosynthetic system works with an impossible efficiency of more than $105 \%$ or $133 \%$, as calculated by bond or reaction energetics, respectively. It is to be restated that the energy for water-heterolysis and pumping protons are still unaccounted in the above calculations. Since the overtly deterministic ZS-KJC paradigm has kinetically incompatible elements, overlooks key observations for photosystem/pigment dependent trace of oxygen transients $(1,3)$, cannot explain the structure-function correlations of PS II and Cyt. $b_{f} f$, and violates fundamental laws of thermodynamics, we deem it imperative that the founding assumptions and equations for Pl-Pp are revamped.

The photolysis of water to liberate oxygen is essentially a four-electron reaction: $2 \mathrm{H}_{2} \mathrm{O} \rightarrow \mathrm{O}_{2}+$ $4 \mathrm{H}^{+}+4 \mathrm{e}^{-}$(or, in an alkaline/aprotic ambiance, $4 \mathrm{OH}^{-} \rightarrow \mathrm{O}_{2}+2 \mathrm{H}_{2} \mathrm{O}+4 \mathrm{e}^{-}$)

Since the emission of one electron requires at least one photon, it would require a minimum of four photons to give water. Breaking of one $\mathrm{O}-\mathrm{H}$ bond would need a photon with an energy quantum of $\sim 464 \mathrm{~kJ} / \mathrm{mol}$; corresponding to the wavelength of $\sim 257 \mathrm{~nm}$. However, we know that 'water-breaking' happens even at visible wavelengths of light. Therefore, it can be inferred that 
the "breaking of $\mathrm{O}-\mathrm{H}$ bonds in water" results later, trailing behind the initial photochemical events. In the murburn view, we would need to tally the energy of photons required for populating the reservoir of electrons, in which case, the theoretical yield would be much lower than the earlier calculations. This means that photosystems 1 \& II merely act as a mechanism for efficient charge-separation or "switches for closing the circuit" function and a significant amount of energy required for photosynthesis is used by the miscellaneous pigments of the chloroplasts. Aquated electrons are known to be spontaneously formed when photo-active species solutions in water are subected to radiations and $\mathrm{O}_{2} / \mathrm{H}_{2} \mathrm{O}$ molecules are ubiquitous within the reaction system. Restricting the above entities' roles or reactivity to specific loci within a highly discretized milieu is merely a deterministic and aesthetic purview, and not a valid theoretical or experimental premise. The facts that the milieu is practically aprotic and copious amounts of DROS are found within chloroplasts $(40)$ or mitochondria $(16,17)$ support the murburn perspective. $\mathrm{O}_{2}$ concentration may range from 50 to $500 \mu \mathrm{M}$ in the water/lipid phases. Also, $\mathrm{O}_{2}$ has a permeability of $10^{7} \mathrm{~nm} / \mathrm{s}$ across phospholipid membranes and its diffusion coefficient in water is $10^{10} \mathrm{~nm}^{2} / \mathrm{s}$, which is an order higher than PQ (41) and two orders higher than PC (42). The PQs of various lengths are present not to primarily serve as mobile agents (as earlier believed) but they are to be seen as 'redox-sponges' packed in the membranes so as to present the reactive heads at different loci of the membrane. Since small oxygen-centered entities would have a linear coverage of $\sim 10^{1}$ to $10^{3} \AA / \mu$ s at the lipid-water interface, the relay of charges across various redox centers greater than $10 \AA$ and/or within $10^{-8}$ to $10^{-3}$ second timescales could also be achieved by murburn equilibriums. Via such equilibriums or direct interactions, the electrondeficient $\mathrm{P}_{680^{+}}$and $\mathrm{P} 700^{+}$could recycle as per the following bimolecular scheme [[Since $\mathrm{OH}^{-}$ $\rightarrow{ }^{*} \mathrm{OH}+\mathrm{e}_{\mathrm{aq}}(446.3 \mathrm{~kJ} / \mathrm{mol}) ; \mathrm{H}_{2} \mathrm{O} \rightarrow \mathrm{H}_{2} \mathrm{O}^{+*}+\mathrm{e}_{\mathrm{aq}}\left(\approx^{*} \mathrm{OH}+\mathrm{H}^{+}+\mathrm{e}_{\mathrm{aq}}=525.3 \mathrm{~kJ} / \mathrm{mol}\right)$; and $\mathrm{P} 700^{+} / \mathrm{P} 680^{+}+\mathrm{e}_{\mathrm{aq}} \rightarrow \mathrm{P} 700 / \mathrm{P} 680(-491 /-561 \mathrm{~kJ} / \mathrm{mol})$, as calculable from the diverse values of redox potentials ranging from 900 to $1300 \mathrm{mV}$ for PS II and 400 to $470 \mathrm{mV}$ for PS I $(43,44)]$ :

$\mathrm{P} 680^{+}+\mathrm{OH}^{-}\left(\mathrm{H}_{2} \mathrm{O}\right) \rightarrow \mathrm{P} 680+{ }^{*} \mathrm{OH}\left({ }^{*} \mathrm{OH}+\mathrm{H}^{+}\right)\left(\Delta_{\mathrm{r}} G_{\mathrm{aq}}^{\mathrm{o}}=-114.7\right.$ or $\left.-35.7 \mathrm{~kJ} / \mathrm{mol}\right)$

$\mathrm{P} 700^{+}+\mathrm{OH}^{-} \rightarrow \mathrm{P} 700+{ }^{*} \mathrm{OH}\left(\Delta_{\mathrm{r}} G_{\mathrm{aq}}^{\mathrm{o}}=-44.7 \mathrm{~kJ} / \mathrm{mol}\right)$

Though hydroxide ion is available at micromolar concentration and its small size, favorable anionic charge and greater mobility in hydrophobic/restricted dimensions (45) would enable a kick-start of the photosynthetic system. Thereafter, the energy released could enable heterolysis of water (which could enable facile cycling of PS I) and hydroxyl radicals formed can react 
among themselves and mediate phosphorylations to give other DROS (peroxide/superoxide), which could subsequently evolve oxygen and heat in steady state. In such scenarios, other DROS like superoxide could also serve as an efficient electron donor to the electron-discharged photosystems and this would be a far more facile event than the extant mechanistic proposals. Singlet oxygen could also be formed in chloroplasts (46), and its reaction with systemic components could also generate peroxide (47), which could be utilized for its redox equivalents. The above proposals based in probabilistic interactions are supported by analyses of structures of two key protein complexes- PS II \& Cyt. $b_{6} f$ (Figure 3) $(34,35)$. The prorteins show multiple grooves and channels (both in trans-membrane and lumen domains), with many redox centers that could be accessed easily by solvent/DROS. This 'porous' facet is not expected if the electron transfers occurred purely via Marcus model or if proton pumps were involved. Further, the presence and non-deterministically positioned and non-covalently held multitudes of pigments across the two proteins call for a stochastic scheme. (Also, why should carotenoids and chlorophylls be present in the trans-membrane region of Cyt. $b_{6} f$ ?) While the extant paradigm does not offer any explanation for the presence of PS II's extra-membrane bulbous extension into the lumen, the murburn proposal sees this structure as low-affinity binding and presentation facets for ADP. The six ADP binding sites each revealed per PS II and Cyt. $b_{6} f$ monomers (Figure 3) have dissociation constants in the range of $10^{-5}$ to $10^{-3} \mathrm{M}$, signifying that binding is relevant at the physiological ADP concentration of $\sim 2.5 \times 10^{-4} \mathrm{M}(48)$. The in silico binding methodology we adopted (16) and data/loci obtained are validated by the non-binding of the negative control molecule of $\mathrm{NADP}^{+}$with both proteins even at $\Delta \mathrm{G}=-1 \mathrm{kcal} / \mathrm{mol}\left(\mathrm{K}_{\mathrm{d}} \sim 10^{-1} \mathrm{M}\right)$.

The balanced reaction of Pl-Pp can be minimaly represented in the murburn scheme by:

$4 \mathrm{OH}^{-}+3 \mathrm{NADP}^{+}+\mathrm{ADPOH}+\mathrm{POH}+\mathrm{H}^{+} \rightarrow \mathrm{O}_{2}+3 \mathrm{NADPH}+\mathrm{ADPOP}+\mathrm{H}_{2} \mathrm{O}+\mathrm{H}_{2} \mathrm{O}_{2} ; h v>1847.1 \mathrm{~kJ} / \mathrm{mol}$ It can be seen that the murburn reaction model correctly approximates the cost of breaking four $\mathrm{O}-\mathrm{H}$ bonds and obligatorily involves the formation of water and production/utilization of DROS. Also, addition of extraneous protons would drive the equilibrium to the right [explaining Jagendorf's experiment, earlier mistaken as a support of the chemiosmostic paradigm (2)]. Further, while 10 reactants are used, only 6 products are formed; which justifies the murburn homeostatic principle based on colligative properties (33). Since NADPH and ATP are the energetically useful products, the pseudo-yield of the murburn model can be conservatively 
taken as $\sim 38 \%[[((3 \times 220)+(1 \times 36)) /(1847) \approx 38 \%]]$. This is because the peroxide formed may also enable further peroxidative phosphorylation (33). As explained earlier for mitochondrial respiration $(16,17,33)$, it must be remembered that the above stoichiometry is purely a theoretical projection, and the actual values determined in experiments would be subjected to multiple variables and statistical averages, owing to DROS dynamics.

CRAS-ZS-KJC paradigm was mooted when the structure of membrane complexes were not known and when it was believed that DROS were purely 'agents of chaos and toxic wastes'. With available structural insights, we can see that the assumptions/explanations of double hits/misses and the constants used to fit the periodicity simulation (30), Q-cycle, the protrusions of proteins, etc. do not offer relevant mechanistic correlations within the CRAS-ZS-KJC perspective. It is questionable that water participates in the reaction only at MnComplex of PS II, particularly when the crystal structure shows that the MnComplex is located just outside the trans-membrane region and is well-buried within the protein. It cannot be envisaged how this catalyst would access a steady supply of water molecules and hold on to them. It is known from in vitro studies that species that cannot be foreseen to bind two molecules of $\mathrm{H}_{2} \mathrm{O}$ like $\mathrm{ZnO} / \mathrm{WSe}_{2}$ (49) or simple Mn-porphyrin based systems (50) could also mediate photocatalytic watersplitting to release $\mathrm{O}_{2}$. Plants can grow, photosynthesize and evolve oxygen in manganesedeficient stature (51), albeit inefficiently. The common "money plants/pothos/devil's vine" (genus Epipremnum) are typical examples. Murburn perspective considers the MnComplex of PS II as a feature that could enable efficient stabilization or equilibriation of excess aquated electrons $\left(\mathrm{e}_{\mathrm{aq}}\right.$ ) and/or DROS. Though MnComplex could enhance oxygenesis, the discrete and delocalized DROS chemistry could also result in oxygen formation, which could explain Emerson's observation. A structure-function analogy could be found in the fungal enzyme chloroperoxidase (CPO), which works via diffusible radicals $(9,26)$. CPO also has a manganese ion, besides the heme-thiolate cofactor. When the fungus is grown in Mn-deficient medium, the CPO formed is active (52), although turnovers are lowered because the enzyme deactivates quicker. Manganese has a highly variable valency (a theoretical range of +7 to -3 ) and it could 'save' PS II Complex from the highly variable redox potentials of the ambience. Also, MnComplex could be an efficient oxidase-peroxidase (akin to the 'turbo logic of Complex IV' in mOxPhos $(16,17,26)$, which could explain the enhanced oxygen production at this locus. 
Structure of the NDH complex and supercomplexes support the murburn model: The extended trans-membrane foot of the NDH protein with a bulbous arm incorporating three FeS proteins (of which the basal FeS is $>2 \mathrm{~nm}$ away from the trans-membrane region) (53) supports a "recruiter" function for this protein. That is- the trans-membrane region serves as an efficient hub for other complexes to come together spatially (54) and optimize DROS dynamics, quite similar to the mitochondrial system $(16,17)$. In the murburn purview, NDH's aqueous-phase arm would enhance one-electron species' lifetimes by 'sinking effect' of the Fe-S centers.

The murburn model could potentially explain the broad action spectrum of PI-Pp: The extant explanation for Pl-Pp states that only PS I and PS II emit electrons when excited at 700 and $680 \mathrm{~nm}$ respectively. However, it is well-known that photosynthesis occurs in the whole visible region of the spectrum of $400-700 \mathrm{~nm}$ (albeit relatively lesser at $500-600 \mathrm{~nm}$ ), with a quantum yield varying from $\sim 0.05$ to $\sim 0.1$ (for $\mathrm{O}_{2}$ liberation or $\mathrm{CO}_{2}$ fixation) (55). In any photoassisted reaction, we must have congruent correlation of light absorption, rate of photosynthesis, and the quantum yield. The non-congruence of the action spectrum with respect to the electron emission features of chlorophyll $a$ is inexplicable within the extant purview. The murburn explanation for Pl-Pp (Figure 2) proposes that all pigments, even within the light harvesting complex (LHC), could absorb photons and emit electrons at discrete wavelengths, forming $\mathrm{e}_{\mathrm{aq}}$ and DROS which could be further stabilized, modulated and utilized by the multiple redox active agents within the thylakoid machinery. We perceive that PS I and PS II give an efficient "chargeseparation and unidirectional switch logic" to regulate the overall electrons' (that are received from several individual photoactive pigments) flow, thereby explaining Emerson's observations in Pl-Pp.

Summation: This manuscript offers a fundamentally new framework for interpreting some wellknown reproducible observations like Emerson's effect, which are crucial for explaining the physiology of oxygenic photosynthesis. Since the fundamental redox metabolic logic of mXM, mOxPhos and Pl-Pp have many similarities (Table 1) and since the mechanism of XM (occurring at microsomes of cytoplasm) and OxPhos (occurring in mitochondria and periplasm) were better explained with murburn concept $(12,17)$, we propose that the same purview can be applied in the 
chloroplastid processes also. In this regard, we urge explorations beyond the conventional ideas that- (i) photoelectric emissions occur only at chlorophyll $a$ of PS I \& PS II, (ii) oxygen evolution occurs only at MnComplex accompanying PS II, (iii) there exist intricate electron transport chains (ETC) within chloroplasts and electron mobilization and transfer occur only via the Marcus outer sphere model, (iv) free electrons/protons have 'high-energy' stature in physiological realms, and (v) DROS are mere toxic wastes in cells. In alignment with known principles of chemico-physics, we propose that photo-excitation of diverse pigments across 400$700 \mathrm{~nm}$ lead to the formation of aquated electrons and DROS at multiple loci within the thylakoids (Figure 2). These species interact with various proteins, pigments, and other redoxactive molecules to facilitate the formation of NADPH, molecular oxygen and ATP. Ockham's razor favors the murburn perspective of unordered, bimolecular and stochastic reactions over the CRAS-ZS-KJC model, which is sequential, multi-molecular and deterministic. Since murburn scheme is based in simple thermodynamic principles, the evolutionary transition from anaerobic (example: sulfate or nitrate systems) to aerobic systems (56) would have been facile. Our proposal for murburn equilibriums involvement is also supported by miscellaneous facts such as(i) electron transfers in chloroplasts are noted to violate the Z-scheme format (57) and a variety of molecules can interact with the 'ETC' to take and give electrons (58), (ii) the spatial distribution and stoichiometry of chloroplast proteins/complexes do not support the extant paradigm but agrees with murburn model $(6,59)$, (iii) phosphate and magnesium (which are essential for photophosphorylation) also inhibit oxygenesis by chloroplasts (29), (iv) leaves that show high rates of photosynthesis have low life spans (60), and (v) diverse marine algae show Emerson effect when supplemented with wavelengths of light corresponding to absorption spectra of accessory pigments (3). It is projected that the new outlook would afford us a greater understanding on the evolution of life, fuel research in photosynthesis, provide deeper insights on plant physiology, and facilitate green processes to harness solar energy.

Acknowledgments and Disclaimers: The work was powered by Satyamjayatu: The Science \& Ethics Foundation. KMM thanks Dheeratma Kelath (Satyamjayatu) for balancing a key equation used in this work. The authors have no conflict of interests to declare.

\section{References}


1. R. Emerson, R. Chalmers, C. Cederstrand, Some factors influencing the long-wave limit of photosynthesis. Proc. Natl. Acad. Sci. U. S. A. 43, 133-143 (1957).

2. J. G. Voet, D. Voet, Biochemistry (Wiley, New Jersey, ed. 4, 2011).

3. L. R. Blinks, Action Spectra of Chromatic Transients and the Emerson Effect in Marine Algae. Proc. Natl. Acad. Sci. U. S. A. 46, 327-333 (1960).

4. K. M. Manoj, Aerobic Respiration: Criticism of the Proton-centric Explanation Involving Rotary Adenosine Triphosphate Synthesis, Chemiosmosis Principle, Proton Pumps and Electron Transport Chain. Biochem. Insights. 11, 1178626418818442 (2018).

5. Govindjee, On the requirement of minimum number of four versus eight quanta of light for the evolution of one molecule of oxygen in photosynthesis: A historical note. Photosynth. Res. 59, 249-254 (1999).

6. D. I. Arnon, H. Y. Tsujimoto, G. M. Tang, Proton transport in photooxidation of water: A new perspective on photosynthesis. Proc. Natl. Acad. Sci. U. S. A. 78, 2942-2946 (1981).

7. T. H. MAUGH, A New Light on Photosynthesis. Science. 213, 994-996 (1981).

8. K. M. Manoj, Murburn concept: a paradigm shift in cellular metabolism and physiology. Biomol. Concepts. 11, 7-22 (2020).

9. K. Murali Manoj, Chlorinations catalyzed by chloroperoxidase occur via diffusible intermediate(s) and the reaction components play multiple roles in the overall process. Biochim. Biophys. Acta. 1764, 1325-1339 (2006).

10. K. M. Manoj, L. P. Hager, Chloroperoxidase, a janus enzyme. Biochemistry. 47, 2997-3003 (2008).

11. K. M. Manoj, S. K. Gade, L. Mathew, Cytochrome P450 reductase: a harbinger of diffusible reduced oxygen species. PLoS One. 5, e13272 (2010).

12. K. M. Manoj, A. Parashar, S. K. Gade, A. Venkatachalam, Functioning of Microsomal Cytochrome P450s: Murburn Concept Explains the Metabolism of Xenobiotics in Hepatocytes. Front. Pharmacol. 7, 161 (2016).

13. A. Parashar, D. A. Gideon, K. M. Manoj, Murburn Concept: A Molecular Explanation for Hormetic and Idiosyncratic Dose Responses. Dose. Response. 16, 1559325818774421 (2018).

14. K. M. Manoj, Debunking Chemiosmosis and Proposing Murburn Concept as the Operative Principle for Cellular Respiration. Biomed. Rev. 28, 35-52 (2018).

15. K. M. Manoj, D. A. Gideon, V. D. Jacob, Murburn scheme for mitochondrial thermogenesis. Biomed. Rev. 29,73-82 (2018).

16. K. M. Manoj, A. Parashar, V. David Jacob, S. Ramasamy, Aerobic respiration: proof of concept for the oxygencentric murburn perspective. J. Biomol. Struct. Dyn. 37, 4542-4556 (2019).

17. K. M. Manoj, V. Soman, V. David Jacob, A. Parashar, D. A. Gideon, M. Kumar, A. Manekkathodi, S. Ramasamy, K. Pakshirajan, N. M. Bazhin, Chemiosmotic and murburn explanations for aerobic respiration: Predictive capabilities, structure-function correlations and chemico-physical logic. Arch. Biochem. Biophys. 676, 108128 (2019).

18. C. P. Whittingham, Inhibition of Photosynthesis by Cyanide. Nature. 169, 838-839 (1952).

19. N. I. Bishop, J. D. Spikes, Inhibition by cyanide of the photochemical activity of isolated chloroplasts. Nature. 176, 307-308 (1955).

20. S. P. Berg, D. W. Krogmann, Mechanism of KCN inhibition of photosystem I. J. Biol. Chem. 250, 8957-8962 (1975).

21. G. Forti, P. Gerola, Inhibition of photosynthesis by azide and cyanide and the role of oxygen in photosynthesis. Plant Physiol. 59, 859-862 (1977).

22. C. I. Ullrich-Eberius, A. Novacky, E. Ball, Effect of cyanide in dark and light on the membrane potential and the ATP level of young and mature green tissues of higher plants. Plant Physiol. 72, 7-15 (1983).

23. A. Parashar, S. K. Gade, M. Potnuru, N. Madhavan, K. M. Manoj, The curious case of benzbromarone: insight into super-inhibition of cytochrome P450. PLoS One. 9, e89967 (2014).

24. K. M. Manoj, S. K. Gade, A. Venkatachalam, D. A. Gideon, Electron transfer amongst flavo- and hemoproteins: diffusible species effect the relay processes, not protein-protein binding. RSC Adv. 6, 24121-24129 (2016).

25. K. M. Manoj, A. Parashar, A. Venkatachalam, S. Goyal, Satyalipsu, P. G. Singh, S. K. Gade, K. Periyasami, R. S. Jacob, D. Sardar, S. Singh, R. Kumar, D. A. Gideon, Atypical profiles and modulations of heme-enzymes catalyzed outcomes by low amounts of diverse additives suggest diffusible radicals' obligatory involvement in such redox reactions. Biochimie. 125, 91-111 (2016). 
26. K. M. Manoj, S. Ramasamy, A. Parashar, D. A. Gideon, V. Soman, V. D. Jacob, K. Pakshirajan, Acute toxicity of cyanide in aerobic respiration: Theoretical and experimental support for murburn explanation. Biomol. Concepts. 11, 32-56 (2020).

27. A. Parashar, A. Venkatachalam, D. A. Gideon, K. M. Manoj, Cyanide does more to inhibit heme enzymes, than merely serving as an active-site ligand. Biochem. Biophys. Res. Commun. 455, 190-193 (2014).

28. K. Wallace, Challenging the current paradigm of the chemiosmotic theory for cyanide toxicity. Toxicology. 432, 152377 (2020). [Editorial foreword on an article by Manoj KM, Soman V. Classical and murburn explanations for acute toxicity of cyanide in aerobic respiration: A personal perspective. Toxicology 2020;432:152369.]

29. N. M. Bazhin. Standard and transformed values of gibbs energy formation for some radicals and ions involved in biochemical reactions. Arch. Biochem. Biophys. 686, 108282.

30. Forbush, B. Kok, M. P. Mcgloin, Cooperation of charges in Photosynthetic O2 evolution-II. Damping Of Flash Yield Oscillation, Deactivation. Photochem. Photobiol. 14, 307-321 (1971).

31. S.C. Huber, Effect of $\mathrm{pH}$ on chloroplast photosynthesis: Inhibition of $\mathrm{O}_{2}$ evolution by inorganic phosphate and magnesium. Biochem. Biophys. Acta. 545,131-140 (1979).

32. J. Shen, Y. Zeng, X. Zhuang, L. Sun, X. Yao, P. Pimpl, L. Jiang, Organelle pH in the Arabidopsis endomembrane system. Mol. Plant. 6, 1419-1437 (2013).

33. K. M. Manoj, N. M. Bazhin, Murburn precepts of aerobic respiration. https://osf.io/hx4p9 (2019).

34. M. Suga, F. Akita, K. Yamashita, Y. Nakajima, G. Ueno, H. Li, T. Yamane, K. Hirata, Y. Umena, S. Yonekura, L.-J. Yu, H. Murakami, T. Nomura, T. Kimura, M. Kubo, S. Baba, T. Kumasaka, K. Tono, M. Yabashi, H. Isobe, K. Yamaguchi, M. Yamamoto, H. Ago, J.-R. Shen, An oxyl/oxo mechanism for oxygen-oxygen coupling in PSII revealed by an X-ray free-electron laser. Science. 366, 334-338 (2019).

35. G. Kurisu, H. Zhang, J. L. Smith, W. A. Cramer, Structure of the cytochrome b6f complex of oxygenic photosynthesis: tuning the cavity. Science. 302, 1009-1014 (2003).

36. N. Cox, D. A. Pantazis, W. Lubitz, Current Understanding of the Mechanism of Water Oxidation in Photosystem II and Its Relation to XFEL Data. Annu. Rev. Biochem. 89, 19.1-19.26 (2020).

37. A. B. Hope, P. Valente, D. B. Matthews, Effects of $\mathrm{pH}$ on the kinetics of redox reactions in and around the cytochromebf complex in an isolated system. Photosynth. Res. 42, 111-120 (1994).

38. S.N. Datta, J. Sudhamsu, A. Pandey, Theoretical determination of the standard reduction potential of plastocyanin in vitro. J. Phys. Chem. B. 108, 8007-8016 (2004).

39. R. A. Alberty, Thermodynamics of Biochemical Reactions (Wiley, New Jersey, 2003).

40. J. D. Woodson, M. S. Joens, A. B. Sinson, J. Gilkerson, P. A. Salome, D. Weigel, J. A. Fitzpatrick, J. Chory, Ubiquitin facilitates a quality-control pathway that removes damaged chloroplasts. Science. 350, 450-454 (2015).

41. M. Blackwell, C. Gibas, S. Gygax, D. Roman, B. Wagner, The plastoquinone diffusion coefficient in chloroplasts and its mechanistic implications. Biochim.Biophys. Acta. 1183, 533-543 (1994).

42. D. G. Sanderson, L.B. Anderson, E. L.Gross, Determination of the redox potential and diffusion coefficient of the protein plastocyanin using optically transparent filar electrodes. Biochim. Biophys. Acta. 852, 269-278 (1986).

43. A. Nakamura, T. Suzawa, Y. Kato, T. Watanabe. Species dependence of the redox potential of the primary electron donor P700 in photosystem I of oxygenic photosynthetic organisms revealed by spectroelectrochemistry. Plant Cell Physiol. 52, 815-823 (2011).

44. F. Rappaport, M. Guergova-Kuras, P. J. Nixon, B. A. Diner, J. Lavergne.Kinetics and pathways of charge recombination in photosystem II. Biochemistry 41, 8518-8527.

45. A. Bankura, A. Chandra, Hydroxide ion can move faster than an excess proton through one-dimensional water chains in hydrophobic narrow pores. J. Phys. Chem. B. 116, 9744-9757 (2012).

46. Krieger-Liszkay, Singlet oxygen production in photosynthesis. J. Exp. Bot. 56, 337-346 (2005).

47. S. A. Khorobrykh, M. Karonen, E. Tyystjarvi, Experimental evidence suggesting that $\mathrm{H}_{2} \mathrm{O}_{2}$ is produced within the thylakoid membrane in a reaction between plastoquinol and singlet oxygen. FEBS Lett. 589, 779-786 (2015).

48. S. Holfgrefe, J. E. Backhausen, C. Kitzmann, R. Scheibe, Regulation of Steady-State Photosynthesis in Isolated Intact Chloroplasts under Constant Light: Responses of Carbon Fluxes, Metabolite Pools and EnzymeActivation States to Changes of Electron Pressure. Plant Cell Physiol. 38, 1207-1216 (1997).

49. F. Hu, L. Tao, H. Ye, X. Li, X. Chen, $\mathrm{ZnO} / \mathrm{WSe}_{2}$ vdW heterostructure for photocatalytic water splitting. $J$. Mater. Chem. C. 7, 7104-7113 (2019).

50. I. Willner, W. E. Ford, J. W. Otvos, M. Calvin, Method of water photolysis. European Patent- EP0040983A1. (1981). https://patentimages.storage.googleapis.com/47/05/fa/0800283f823783/EP0040983A1.pdf 
51. Janpen, N. Kanthawang, C. Inkham, F. Y. Tsan, S. R. Sommano, Physiological responses of hydroponicallygrown Japanese mint under nutrient deficiency. PeerJ. 7, e7751 (2019).

52. X. Wang, H. Tachikawa, X. Yi, K. M. Manoj, L. P. Hager, Two-dimensional NMR Study of the Heme Active Site Structure of Chloroperoxidase. J. Biol. Chem. 278, 7765-7774 (2003).

53. X. Pan, D. Cao, F. Xie, F. Xu, X. Su, H. Mi, X. Zhang, M. Li, Structural basis for electron transport mechanism of complex I-like photosynthetic NAD(P)H dehydrogenase. Nat. Commun. 11, 610 (2020).

54. N. S. Yadav, D. A. Semchonok, L. Nosek, R. Kouril, G. Fucile, E. J. Boekema, L. A. Eichacker, Supercomplexes of plant photosystem I with cytochrome b6f, light-harvesting complex II and NDH. Biochim. Biophys. acta. Bioenerg. 1858, 12-20 (2017).

55. S. W. Hogewoning, E. Wientjes, P. Douwstra, G. Trouwborst, W. van Ieperen, R. Croce, J. Harbinson, Photosynthetic quantum yield dynamics: from photosystems to leaves. Plant Cell. 24, 1921-1935 (2012).

56. M. Leslie, On the Origin of Photosynthesis. Science. 323, 1286-1287 (2009).

57. Barber, Short-circuiting the Z-scheme. Nature. 376, 388-389 (1995).

58. J. Tschörtner, B. Lai, J. O. Krömer, Biophotovoltaics: Green Power Generation From Sunlight and Water . Front. Microbiol. 10, 866 (2019).

59. S. D. McKenzie, I. M. Ibrahim, U. K. Aryal, S. Puthiyaveetil, Stoichiometry of protein complexes in plant photosynthetic membranes. Biochim. Biophys. Acta - Bioenerg. 1861, 148141 (2020).

60. S. Matsuki, T. Koike, Comparison of leaf life span, photosynthesis and defensive traits across seven species of deciduous broad-leaf tree seedlings. Ann. Bot. 97, 813-817 (2006).

\section{TABLES \& FIGURES}

Table 1: Similarities in the reaction schematics of $\mathrm{mXM}, \mathrm{Pl}-\mathrm{Pp}$ and $\mathrm{mOxPhos}$ systems

\begin{tabular}{|c|c|c|c|c|c|c|c|c|c|c|c|}
\hline \multirow{3}{*}{$\begin{array}{c}\text { System } \\
m X M\end{array}$} & \multicolumn{5}{|c|}{$\begin{array}{c}\text { Reaction components } \\
\text { (Nucleotide, Substrate, O-source) }\end{array}$} & & \multicolumn{5}{|c|}{ Products and outcomes ${ }^{*}$} \\
\hline & NADPH & + & $\mathrm{R}$ & + & $\mathrm{O}_{2}$ & $\rightarrow$ & $\mathrm{NADP}^{+}$ & + & $\mathrm{ROH}+\mathrm{H}_{2} \mathrm{O}_{2}+\mathrm{H}_{2} \mathrm{O}$ & + & Heat \\
\hline & & & Uncoupled & & & $\rightarrow$ & $\mathrm{NADP}^{+}$ & + & $\mathrm{ROH} \downarrow+\mathrm{H}_{2} \mathrm{O}_{2} \uparrow+\mathrm{H}_{2} \mathrm{O} \uparrow$ & + & Heat个 \\
\hline \multirow{2}{*}{$P I-P p$} & NADP $^{+}$ & + & ADP \& Pi & + & $\mathrm{H}_{2} \mathrm{O}+\mathrm{hv}$ & $\rightarrow$ & NADPH & + & $\mathrm{ATP}+\mathrm{H}_{2} \mathrm{O}_{2}+\mathrm{O}_{2}$ & + & Heat \\
\hline & & & Uncoupled & & & $\rightarrow$ & NADPH $\downarrow$ & + & ATP $\downarrow+\mathrm{H}_{2} \mathrm{O}_{2} \uparrow+\mathrm{O}_{2} \uparrow$ & + & Heat个 \\
\hline \multirow{2}{*}{ mOxPhos } & $\mathrm{NADH}$ & + & ADP \& Pi & + & $\mathrm{O}_{2}$ & $\rightarrow$ & $\mathrm{NAD}^{+}$ & + & $\mathrm{ATP}+\mathrm{H}_{2} \mathrm{O}_{2}+\mathrm{H}_{2} \mathrm{O}$ & + & Heat \\
\hline & & & Uncoupled & & & $\rightarrow$ & $\mathrm{NAD}^{+}$ & + & $\mathrm{ATP} \downarrow+\mathrm{H}_{2} \mathrm{O}_{2} \uparrow+\mathrm{H}_{2} \mathrm{O} \uparrow$ & + & Heat个 \\
\hline
\end{tabular}

*For a particular reaction system, the first row details the productive reaction whereas the second row gives the uncoupled reaction, with or without the addition of uncouplers/substrates. Upward and downward arrows indicate enhancement or lowering, respectively. 


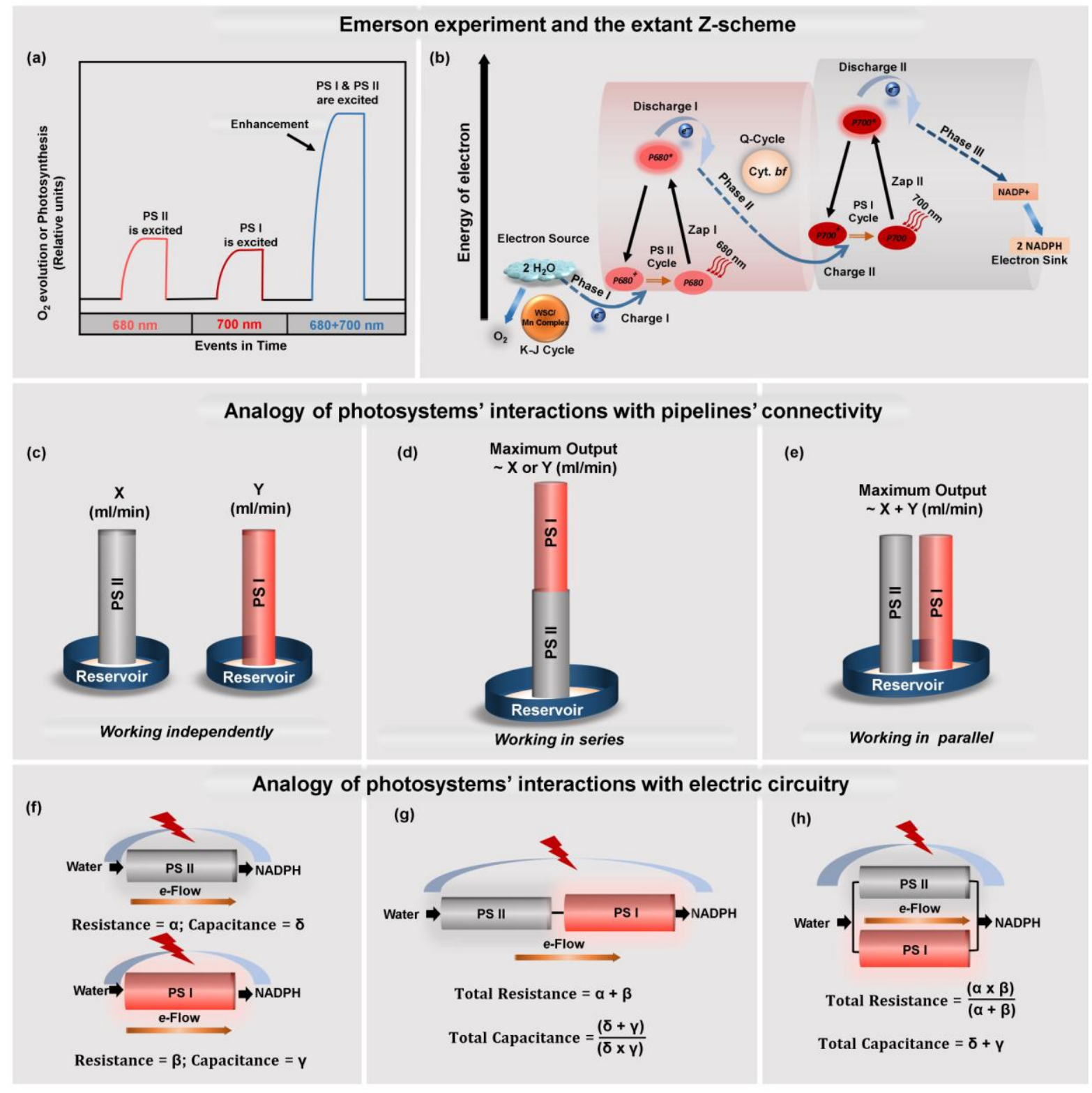

Figure 1: (a) Emerson experiment, (b) Z-scheme of electron transfer, (c-e) Comparing the photosystems to fluid pipelines If a positive pressure existed in the reservoir so that the fluid flows $(\approx$ electron flow) out, the series connectivity would maximally give a flow rate (fluid collected in given time, equivalent to oxygen collected) of either PS I or PS II only (depending on which system is rate limiting). But a parallel plumbing can give a total flow more than the individual photosystems. $(\boldsymbol{f}$ - $\boldsymbol{h})$ Comparing electron flow through photosystems with electrical circuitry. If light served to generate an emf, the electron flow within the chloroplast redox machinery could be effectively compared to an electrical circuit in steady state. In this case, the enhanced electron flow can be derived with a parallel connectivity of the conducting and charge-releasing components of the circuitry. (Refer text for details.) 


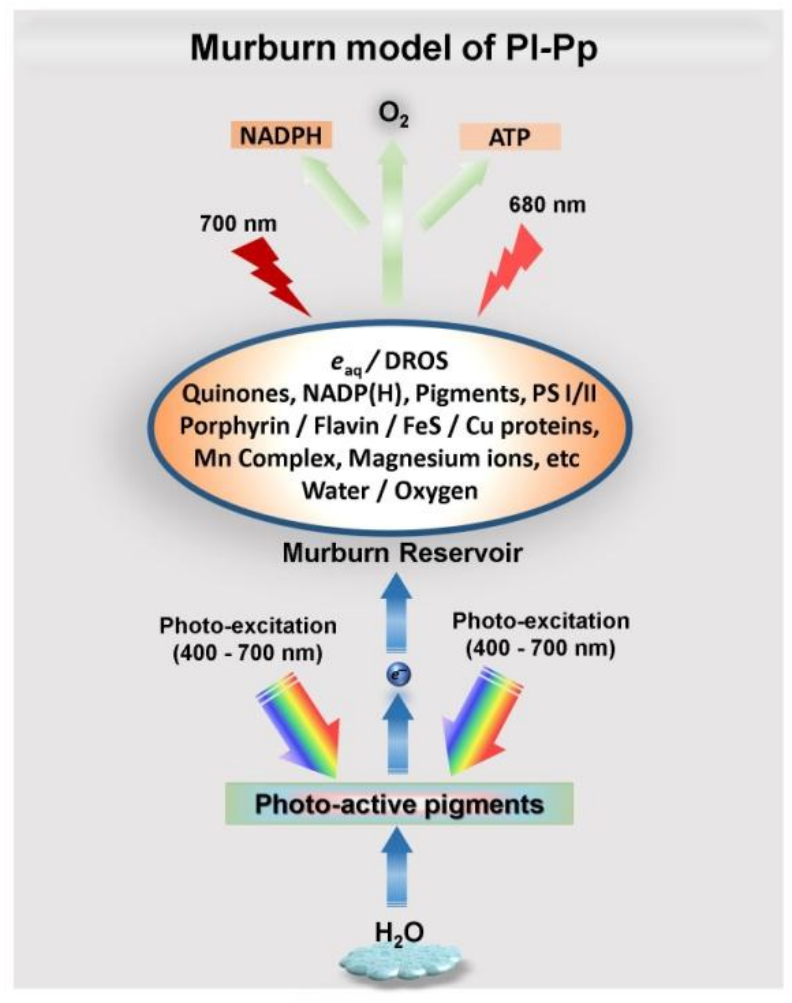

Figure 2: The murburn model of Pl-Pp. Light (400 to $700 \mathrm{~nm}$ ) excites photo-active pigments to eject electrons, which in turn get aquated or are taken up by various components within the milieu. PS I and II enable efficient charge separation and $2 \mathrm{e}$ sinking with the aid of molecules like pheophytin and quinones. Cyt. $b f \&$ PC reset a one-electron paradigm from the electrons lost to membrane phase whereas PC, $\mathrm{NADP}^{+}, \mathrm{Fd}$, etc. capture/recycle electrons in the aqueous phase, enabling NADPH formation. DROS aid ADP phosphorylation or react among themselves to give oxygen and heat, and oxygen is also formed by other mechanisms initiated by PS I \& II. Various participating entities within the murburn reservoir are connected by interactive one-electron equilibriums and there are no "high energy electrons/protons" and very few affinity-based electron transfers. In toto, the overall equation is: $4 \mathrm{OH}^{-} \rightarrow \mathrm{O}_{2}+2 \mathrm{H}_{2} \mathrm{O}+4 \mathrm{e}^{-}$ 

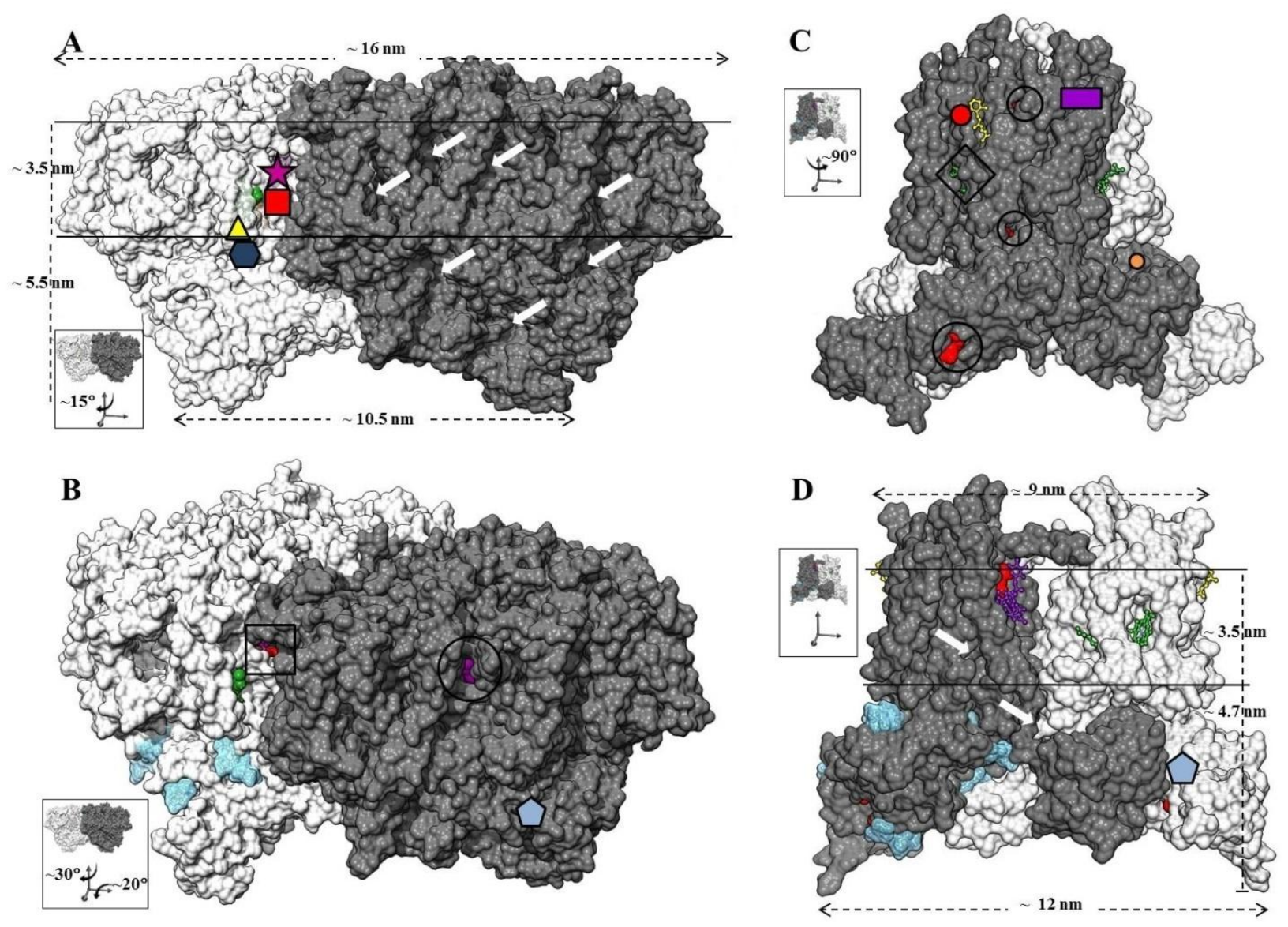

Figure 3: Structure-function correlations of two key membrane protein complexes of thylakoids: Insets show the resting frame of the protein and the degrees of rotation around the respective axes. Horizontal lines demarcate the trans-membrane region. A) Dimeric structure of PS II multi-protein complex (6JLJ). The external dimensions and locations of the key redox centers are shown. On the white-grey monomer, the relative loci of MnComplex (blue-grey hexagon), Tyr161 (yellow triangle), RC (red square) and pheophytin (magenta star) are indicated. $\mathrm{Chlz}_{\mathrm{D} 1}$ (green) is visible near the RC. Samples of crypts and channels are indicated by the white arrows on the black-grey monomer. B) ADP-binding sites on PS II. There are six ADP-binding sites per monomer $\left(\mathrm{K}_{\mathrm{d}}\right.$ ranging from $\sim 40 \mu \mathrm{M}$ to $\left.\sim 2.6 \mathrm{mM}\right)$, three of which are visible (shown as sky-blue ADP molecules docked on to the white-grey monomer) on the presented face and a distinct ADP-binding site is depicted with a sky-blue pentagon on the black-grey monomer. Channels accessing the RC chlorophylls (red and orange) on the white-grey monomer and pheophytin (magenta) on the black-grey monomer are shown within a square and a circle respectively. Pheophytin (magenta) on the black-grey monomer is accessible through channel and is shown encircled. $\mathrm{Chlz}_{\mathrm{D} 1}$ is also visible and shown in green. C) The structure of Cyt. $\boldsymbol{b}_{\boldsymbol{\sigma}} \boldsymbol{f}$ and location of its redox centers (1VF5). Except FeS center (orange circle), all other redox elements are accessible through channels. Representations are: four hemes: red (encircled at three locations and one hidden behind the face is represented with a red-filled circle), $\beta$-carotene: yellow, chlorophyll: green (visible through channel depicted in a rhombus) and quinone: purple-filled rectangle. D) ADP binding sites on Cyt. $\boldsymbol{b}_{\boldsymbol{f}} \boldsymbol{f}$. Six ADP binding clusters ( $\mathrm{K}_{\mathrm{d}}$ ranging from $\sim 160 \mu \mathrm{M}$ to $\sim 1.5 \mathrm{mM}$ ) are present, of which four are shown docked (sky blue space-filled ADP molecules) on the black-grey monomer. A distinct locus is shown as blue pentagonal representation on the white-grey monomer. Quinone (purple) and chlorophyll (green) located in a wide cavity can also be seen along with carotenoids (yellow) in the trans-membrane region. 